\title{
Résilience des systèmes urbains : proposition d'un cadre méthodologique pour répondre aux besoins des exploitants
}

\section{Resilience of urban systems: a proposal for a methodological framework dedicated to the operators' needs}

\author{
Michaël Gonzva ${ }^{1,2}$, Bruno Barroca ${ }^{2}$, Damien Serre ${ }^{3}$ \\ ${ }^{1}$ SYSTRA, Direction Innovation, France, mgonzva@systra.com \\ ${ }^{2}$ Université Paris-Est Marne-la-Vallée, Lab'Urba (Laboratoire d'Urbanisme - Département Génie Urbain), France, \\ bruno.barroca@u-pem.fr \\ ${ }^{3}$ Université d'Avignon et des Pays du Vaucluse, UMR ESPACE 7300 CNRS, France, damien.serre@univ-avignon.fr
}

\begin{abstract}
RÉSUMÉ. L'objectif de cet article consiste à présenter une démarche méthodologique de résilience développée depuis une dizaine d'années dans le but de prendre en compte la complexité des systèmes d'infrastructures critiques urbaines dans un contexte de maîtrise et gestion des risques. Cette complexité est appréhendée sous l'angle de l'omniprésence des interdépendances, communément démontrées comme vecteur de propagation des perturbations. La démarche méthodologique a été appliquée à plusieurs systèmes urbains et a été testée dans le but d'y intégrer la complexité et de produire de la connaissance nécessaire à la formalisation de l'action en faveur de la gestion des risques.

ABSTRACT. The objective of this paper consists in presenting a resilience methodological approach developed since decade with the aim of taking into account the complexity of urban critical infrastructures in a context of risks management. This complexity is tackled according to the omnipresence of interdependences, collectively demonstrated as vector of initiating and spreading disruptions. The methodological approach is applied to several urban systems and tested with the objective of integrating the complexity and producing required knowledge the actions in favour of the risk management.
\end{abstract}

MOTS-CLÉS. Risques, gestion, méthodologie, résilience, opérationnel, systémique, critique.

KEYWORDS. Risks, management, methodology, resilience, operational, holistic, critical.

\section{Introduction}

Indépendamment de leurs spécificités géographiques, sociales ou économiques, toutes les villes font face à des risques de typologie différente et d'intensité variable. Malgré la diversité des risques il existe des traits communs essentiellement liés à la spécificité des territoires urbains fondamentalement marqués par leur complexité. Leur maîtrise et gestion soulèvent des interrogations théoriques liées à la compréhension des phénomènes et des défis opérationnels quant aux actions concrètes de mitigation à mettre en œuvre. Parmi les éléments-clés de la maîtrise des risques, les infrastructures critiques particulièrement vulnérables sont des éléments essentiels au fonctionnement urbain.

De façon générale au sein des projets de recherche européens [EWE 12; MOW 14], les infrastructures critiques ont été analysées vis-à-vis de leurs fragilités (dysfonctionnements, défaillances de composants, etc.), de leurs dépendances (interactions, rétroactions, etc.) et de leurs services (mode dégradé d'exploitation). La volonté de fiabilisation pour un fonctionnement en mode dégradé est un des résultats qui a amené à sécuriser et à protéger les éléments les plus fragiles. Du côté de la recherche urbaine se développe depuis les années 2000 le concept de résilience. La résilience peut se définir comme la capacité d'un système à absorber le changement, à persister au-delà d'une perturbation et à retrouver rapidement un niveau de service correspondant aux besoins.

L'objectif de cet article consiste à présenter une démarche méthodologique développée depuis une dizaine d'années dans le but de prendre en compte la complexité des systèmes d'infrastructures 
critiques urbaines dans un contexte de maitrise et gestion des risques. Cette complexité est appréhendée sous l'angle de l'omniprésence des interdépendances, communément démontrées comme vecteur de propagation des perturbations. Notre démarche méthodologique a été appliquée à plusieurs systèmes urbains - systèmes de transport public [GON 17], réseaux de gestion des déchets [BER 13], systèmes de digues de protection face aux inondations [SER 05 ; VUI 12] -, et a été testée dans le but d'y intégrer la complexité et de produire de la connaissance nécessaire à la formalisation de l'action en faveur de la gestion des risques.

\section{Synthèse des méthodes dominantes mobilisées pour l'analyse des risques affectant les systèmes urbains}

Dans la vision développée dans cet article, toute démarche d'amélioration de la résilience nécessite au préalable une analyse de la vulnérabilité. Autrement dit, sans prendre le parti théorique de considérer la vulnérabilité comme dimension constitutive de la résilience, l'hypothèse consiste simplement à dire que pour améliorer la résilience d'un système urbain tel qu'un système de transport face à un aléa donné, il est nécessaire mais non suffisant d'en connaître d'abord la vulnérabilité face à cet aléa. La vulnérabilité devenant, en poussant l'idée à l'extrême, le nécessaire diagnostic pour la mise en place, dans un second temps, de stratégies appropriées de résilience. En l'occurrence, une fois connues les vulnérabilités d'un système de transport guidé implanté en souterrain, en surface et en aérien face au risque inondation, la réponse théorique et pratique à apporter ne consiste pas en la résolution une à une de ces vulnérabilités prises comme des obstacles indépendants à la résilience globale du système. La résilience d'un système ne s'accroît pas, en effet, par la réduction voire suppression de l'ensemble de ses vulnérabilités mais revêt un aspect bien plus global qui s'illustre très bien par le fait que diminuer la vulnérabilité d'un système à un endroit donné peut créer ou accroître la vulnérabilité d'un autre système en d'autres temps ou endroit ${ }^{1}$ [NEL 07]. Par conséquent, la démarche d'amélioration de la résilience est bien distincte de la démarche d'analyse de la vulnérabilité. Mais la seconde est nécessaire, quoique non suffisante, à la réalisation de la première.

Dans cet article, par ailleurs, les systèmes urbains sont considérés comme des systèmes de type technico-fonctionnel pour plusieurs raisons [GON 17]. D'une part, ce sont des objets techniques avec un but précis permis par une fonction d'usage et afin de satisfaire un besoin donné. La caractéristique physique et matérielle de ces systèmes à partir de laquelle transitent des flux de tous types (informations, énergie, etc.) en fait donc des objets techniques. D'autre part, ces systèmes ont une caractéristique fonctionnelle: le support physique qui assure les échanges de flux nécessite une organisation globale précise afin que la (ou les) fonction(s) du système soi(en)t assurée(s). La caractéristique technicofonctionnelle est également pertinente car c'est par cette double composante qu'est abordée la résilience [SER 11; SER 15]: technique, afin d'évaluer les dommages matériels directs et indirects provoqués par un aléa sur un système, et fonctionnelle, afin d'évaluer les conséquences sur l'exploitation c'est-à-dire sur le niveau de service que peut fournir le système (niveau nominal, niveau faiblement dégradé, niveau moyennement dégradé, etc.) et l'organisation requise par ce niveau de service (ressources à mobiliser, notamment).

La notion de système est, ainsi, fondamentale dans l'approche menée. Penser système, c'est de fait s'acheminer sur le terrain de la complexité et, réciproquement, analyser la complexité est possible par une approche systémique. Dans ce contexte, la compréhension des systèmes qui constituent les villes peut paraître partielle, avec d'importantes zones d'ombre. Il apparait alors important de distinguer la complexité de la complication afin d'identifier sur quel terrain se place ce travail. La différence fondamentale entre ces deux termes est la suivante [BAR 16] : un objet compliqué peut être simplifié alors que la complexité est un état fondamental qui définit l'objet considéré. Un objet complexe ne doit, par conséquent, pas être simplifié. Dès lors, les systèmes urbains sont des systèmes compliqués

\footnotetext{
1 "Reducing vulnerability in one area creates or increases vulnerability in an-other area or time."
} 
voire hyper compliqués plutôt que des systèmes complexes au sens de la systémique car ils ne présentent pas, par exemple, de phénomènes qui émergent de façon imprévisible dans leur fonctionnement. Néanmoins, le haut degré de complication ou l'hyper complication du fonctionnement, et surtout du dysfonctionnement, d'un système urbain soumis à un aléa nécessite de s'orienter vers les approches offertes par la systémique.

Les systèmes qui constituent les villes sont considérés comme des infrastructures critiques. Leurs caractéristiques principales sont établies clairement [KRO 08] et collectivement identifiées par un grand nombre d'institutions internationales [DHS 2006, PAR 08; PSC 2009]. Structurellement, ils sont composés d'un grand nombre d'éléments qui interagissent entre eux de façon non linéaire [SIM 91] et dans lesquels les relations de cause à effet ne sont pas toujours établies scientifiquement et nécessitent alors une part d'intuitif [SER 05, SER 11]. Pour minimiser cette part d'intuitif qui peut représenter un danger dans la compréhension du fonctionnement et surtout des dysfonctionnements de ces infrastructures critiques, il est possible d'avoir recours respectivement à un modèle fonctionnel et dysfonctionnel du système.

La modélisation fonctionnelle comme moyen d'analyser les risques qui peuvent endommager les systèmes urbains apparaît donc prometteuse pour réduire le haut degré de complication d'ordre technique et organisationnel de ces systèmes car cette haute complication a pour conséquence de rendre la plupart des approches physiques classiques peu voire non efficaces pour comprendre le fonctionnement [ZWI 96, VIL 97] de ces systèmes. Cette situation a alors offert un regain d'intérêt pour un certain type de méthodes pour l'analyse des risques impactant les systèmes urbains : les méthodes issues de la Sûreté de Fonctionnement. La Sûreté de Fonctionnement consiste à connaître, évaluer, prévoir, mesurer et maîtriser les défaillances de systèmes technologiques, tout comme les défaillances humaines, afin de limiter les conséquences de ces défaillances sur la santé, la sécurité des personnes, la productivité et l'environnement [NOY 07]. La Sûreté de Fonctionnement est tout à fait adaptée pour une analyse des défaillances des composants propres à un système urbain lorsque survient un aléa naturel, en s'appuyant sur un support méthodologique diversifié et puissant.

Parmi ces méthodologies, l'Analyse Fonctionnelle pour élaborer un modèle de fonctionnement du système et l'Analyse des Modes de Défaillance et leurs Effets (AMDE) s'avèrent pertinentes et appropriées dans le cas de systèmes urbains touchés par un risque naturel tel qu'une inondation [GON 16]. Premièrement, l'Analyse Fonctionnelle permet une décomposition matérielle et fonctionnelle d'un système en cours de conception ou en fonctionnement. L'Analyse Fonctionnelle consiste alors à recenser, caractériser, ordonner, hiérarchiser et valoriser les fonctions assurées par les composants du système [ZWI 09]. Deuxièmement, une autre méthode, usuellement appliquée consécutivement à l'Analyse Fonctionnelle, est l'Analyse des Modes de Défaillance et de leurs Effets (AMDE). C'est une méthode efficace pour l'analyse des modes de défaillance en permettant de structurer, sous forme de tableaux, les informations sur les dégradations : les pertes de performance, leurs causes, leurs effets [TAL 09]. L'ADME est inductive c'est-à-dire qu'en partant des causes des défaillances et en remontant jusqu'à leurs conséquences, elle considère un à un les composants du système et analyse leurs modes de défaillance.

Ainsi, les méthodes issues de la Sûreté de Fonctionnement font l'objet, depuis une quinzaine d'années, d'applications remarquables sur des systèmes urbains offrant des résultats théoriques et opérationnels. Ces applications concernent en majeure partie l'ambition de connaissances en faveur de la résilience urbaine mais ne s'y résument pas pour autant. En particulier, l'Analyse Fonctionnelle est appliquée à de nombreux systèmes urbains [GON 17] : produits de construction du bâtiment [LAI 00, TAL 06], barrages [PEY 02], routes [VAL 03], systèmes de gestion des déchets [BER 13], systèmes et ouvrages de protection [SER 05, VUI 02], etc. Cette tendance ne s'essouffle guère avec des utilisations en cours de l'Analyse Fonctionnelle dans des projets de recherche (figure 1). 


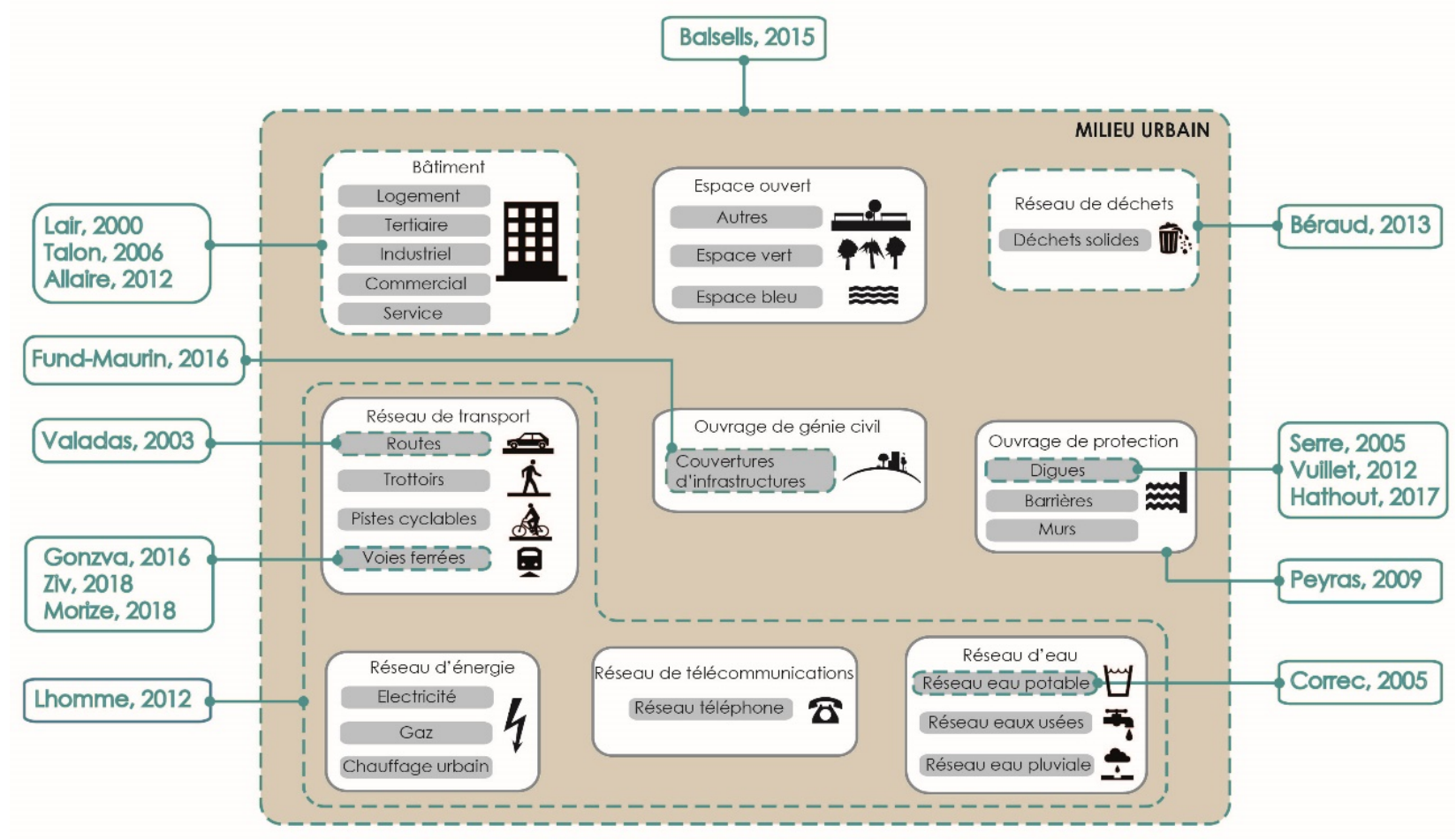

Figure 1. Etat de l'art des applications majeures de l'analyse fonctionnelle issue de la Sûreté de Fonctionnement en génie urbain [GON 17]

\section{Exemple à l'échelle d'une infrastructure critique : les systèmes de transport}

Dans une démarche d'analyse de la vulnérabilité des systèmes de transport, préliminaire nécessaire à une démarche d'amélioration de leur résilience, trois méthodes issues de la Sûreté de Fonctionnement sont mobilisées consécutivement : l'Analyse Fonctionnelle, l'Analyse des Modes de Défaillance et de leurs Effets et les Arbres d'Evènements (figure 2). Cette troisième méthode est utilisée pour représenter les résultats obtenus lors de l'AMDE. Ces Arbres d'Evènements servent dans leur caractéristique causale c'est-à-dire pour formaliser sous forme de graphes d'évènements causaux une séquence d'évènements constituée d'un évènement initiateur et d'une combinaison de défaillances successives.

La démarche méthodologique s'avère, de plus, efficace pour traiter les trois configurations classiques d'implantation des éléments d'un système de transport guidé : en souterrain, en surface et en aérien. Dans le cas du risque inondation, cet aspect est déterminant en matière de vulnérabilité du système.
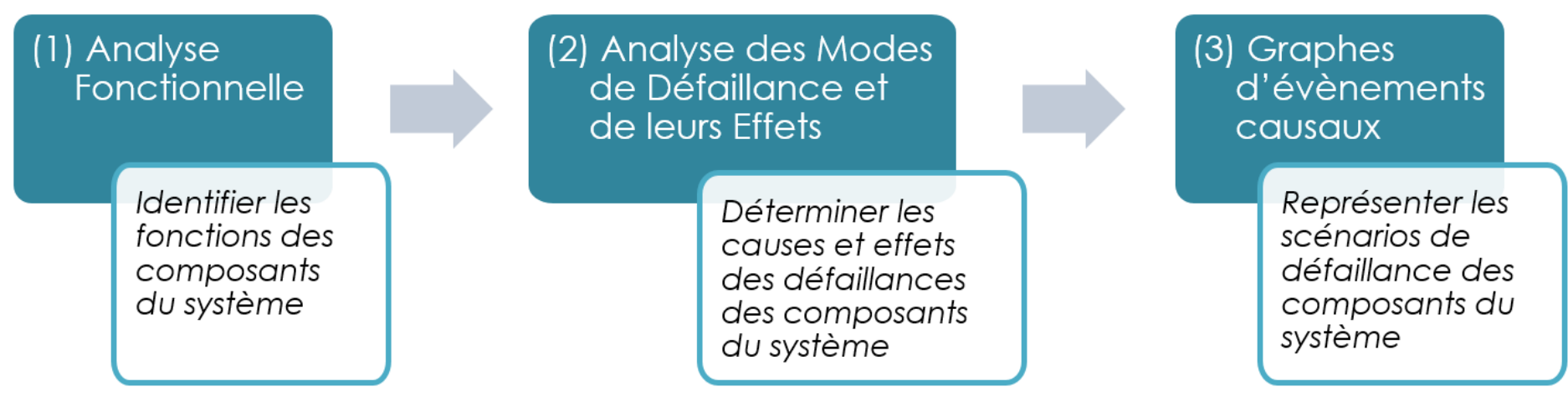

Figure 2. Démarche méthodologique globale de caractérisation de la vulnérabilité d'un système de transport guidé soumis à un risque inondation [GON 17] 
A partir d'une étude statistique sur l'ensemble des scénarios de défaillances par effet domino, une analyse comportementale des composants est réalisée. Trois profils de composants émergent en situation de risque, lorsque le système subit une inondation [GON 17]: les composants majoritairement situés en première position des scénarios de défaillances vont avoir un rôle agressif à l'égard des autres composants dès lors que le système subit une inondation, les composants majoritairement situés en dernière position des scénarios auront un profil sensible, et enfin, les composants majoritairement situés en position intermédiaire des scénarios ont un rôle de diffusion des défaillances.

Ainsi, cette démarche méthodologique globale permet la production des scénarios de défaillances par effet domino des composants du système lorsqu'il est touché par une inondation. En ce sens, elle est bien une démarche d'analyse "par l'intérieur" [GOU 13] de la vulnérabilité des systèmes de transport, c'est-à-dire au travers des mécanismes internes de défaillances entre composants.

\section{Contribution des analyses à l'échelle d'infrastructures critiques à l'amélioration de la résilience urbaine}

Dès lors, deux interrogations se posent. Premièrement, cette démarche méthodologique systémique est-elle applicable à d'autres systèmes urbains, voire à tous les systèmes urbains indépendamment du service délivré (électricité, eau, déchets, etc.) ? Dans ce cas, elle offrirait un cadre d'analyse commun des systèmes urbains sous l'angle des risques et permettrait donc une compréhension cohérente de leur perturbation lorsque survient un aléa naturel. Deuxièmement, dans quelle mesure une analyse individuelle de la vulnérabilité des systèmes urbains à partir d'un tel cadre méthodologique formel peut-elle être un socle de connaissances dans le but d'améliorer la résilience de la ville ? Cette seconde interrogation a un corolaire : quels développements futurs, théoriques et pratiques, de ce cadre méthodologique doivent être réalisés pour renforcer la résilience de la ville à partir de l'amélioration de la résilience de ses systèmes constitutifs ?

Concernant la première interrogation, une raison d'ordre structurel étaye la possibilité de transposition de la démarche à d'autres systèmes urbains. Ces derniers présentent, en effet, pour une majeure partie une architecture du système similaire : un système composé en sous-systèmes, euxmêmes constitués de composants qui peuvent être à leur tour dissociés en éléments plus fins. A chaque niveau de granularité, l'élément peut être considéré comme un système, et dès lors la démarche est potentiellement applicable.

Par exemple, le réseau électrique en France peut être distingué en trois sous-systèmes :

- la production : ce sous-système est constitué notamment des barrages hydrauliques, des centrales nucléaires, des éoliennes, des parcs de panneaux photovoltaïques ;

- le transport et la distribution : ce sous-système est composé entre autres des lignes à haute, moyenne et basse tension et les postes sources associés ;

- la consommation : ce sous-système est constitué de l'ensemble des consommateurs d'électricité, industriels et particuliers.

Il est aisé de constater que chaque composant peut être dissocié à son tour en éléments plus fins. De cette manière, il devient possible de réappliquer la démarche méthodologique globale permettant l'identification des scénarios de défaillances par effet domino des composants. Par ailleurs, la capacité de la démarche à intégrer des niveaux différents d'implantation de composants du système étudié est un atout. En effet, la plupart des systèmes urbains présentent des caractéristiques d'implantation différentes sur l'ensemble de leur infrastructure : éléments positionnés en souterrain (lignes électriques basse tension, câbles de télécommunications, conduites de gaz, dispositifs de collecte pneumatique des déchets, etc.), en surface (postes sources électriques, pylônes de support de lignes électriques, usine de traitement de l'eau, etc.) ou en aérien (lignes électriques haute tension, réservoirs ou châteaux d'eau, 
etc.). La méthodologie permet donc d'inclure dans l'analyse les particularités du territoire urbain sur lequel est ou sera implanté le système urbain étudié : selon le contexte local géographique, météorologique, urbain, la démarche met en avant la configuration d'implantation souterrain, en surface ou aérienne la plus à même de réduire la vulnérabilité. Ainsi, de la même façon que dans le cas d'un système de transport, cette caractéristique est essentielle à intégrer dans les analyses de vulnérabilité et, consécutivement, dans les stratégies d'amélioration de la résilience de ces systèmes.

La seconde interrogation aborde la portée opérationnelle des connaissances générées par un tel cadre méthodologique pour le renforcement de la résilience à l'échelle urbaine. En ce sens, elle oriente les perspectives de travail vers les opérateurs et gestionnaires des systèmes urbains concernés par l'enjeu que représente la gestion complexe de l'ensemble des risques susceptibles de survenir. Le projet européen RAIN (2014-2017) pour Risk Analysis of INfrastructure networks in response to extreme weather a notamment consisté à mener des interviews auprès de 27 opérateurs et gestionnaires européens d'infrastructures critiques issus des secteurs de la distribution d'énergie, du transport ferroviaire et routier, des télécommunications et des services de gestion de crise. Ces interviews mettent en avant les risques naturels mentionnés comme ayant les impacts globaux les plus importants. Les impacts sociaux sont également précisés lorsqu'ils sont significatifs (figure 3). Deux conclusions, étayant la démarche méthodoligque proposée dans cet article, apparaissent dans ce projet. D'une part, les aléas naturels affectant les systèmes urbains sont très divers et nombreux. Les aléas de pluies, de précipitations verglaçantes, de tempêtes venteuses, de tempêtes de neige, et de crues de cours d'eau sont mentionnés comme ayant le plus de conséquences sur les réseaux. Certains aléas, bien qu'affectant peu les opérateurs et gestionnaires interrogés, ont pourtant un impact significatif sur la société qui nécessite de les traiter. Cette diversité d'aléas naturels, même peu fréquents, doit donc faire l'objet d'une gestion et d'une maîtrise des risques qu'ils peuvent générer. D'autre part, les réseaux routiers, les réseaux ferrés et les systèmes de secours font partie des plus touchés par les aléas naturels, notamment du fait que leur fonctionnement peut être pertubé sans endommagements matériels particuliers. Ainsi, la démarche proposée répond pleinement aux besoins métiers concordants, c'est-àdire ceux soulevés par les gestionnaires et exploitant des systèmes urbains en matière d'aléas naturels : assurer une réduction et une maîtrise de l'ensemble des conséquences des aléas naturels par le biais de méthodes, mettre en place des démarches applicables à plusieurs types de systèmes urbains et permettant également d'apporter de la connaissance en matière d'amélioration globale de la résilience urbaine. 


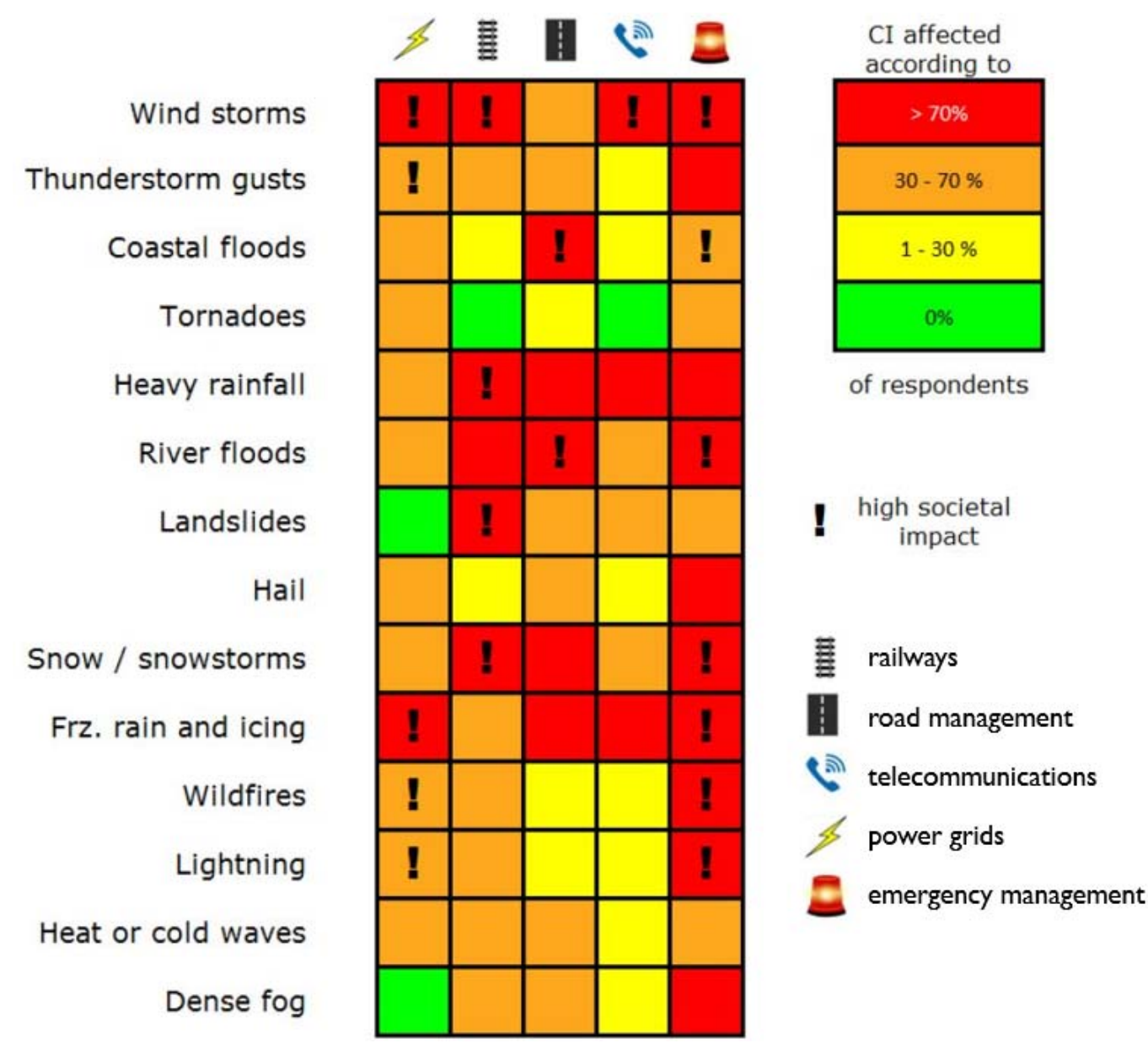

Figure 1. Résultats d'interviews menées dans le cadre du projet européen RAIN auprès de gestionnaires et exploitants européens d'infrastructures critiques ayant à faire face à des risques naturels [GRO 15]

Il semble que ce soit au travers de la continuité d'activité des systèmes urbains que peut se positionner une démarche d'amélioration de leur résilience. En particulier, la capacité à faire fonctionner les systèmes urbains en mode dégradé est une composante importante de la résilience [LHO 10, TOU 14]. En effet, la résilience d'un système est directement liée à son degré de préparation en matière de continuité d'activité lors d'un évènement perturbateur en : réduisant les répercussions de la crise à court, moyen et long terme, en raccourcissant la phase d'inactivité et en facilitant le retour à une activité dite normale [COM 13]. La continuité d'activité donne au concept de résilience une portée collective qui dépasse l'échelle du système.

La démarche méthodologique, une fois appliquée à un système urbain tel que les systèmes de transport guidé face au risque inondation, offre justement des connaissances en faveur de l'élaboration de stratégies de continuité d'activité. Pour illustration dans le cas d'un système de transport souterrain, cette distinction se traduit de la manière suivante :

- les espaces d'accès des gares et les pompes d'épuisement, visant à absorber les infiltrations ou les remontées d'eau ont un profil essentiellement agressif ;

- les câbles situés en gare et le long des voies ferrées ont un profil principalement sensible ;

- le tunnel a un comportement important de diffuseur de risque.

Ces profils de comportement de composants lors de l'occurrence d'aléas naturels interrogent et guident également les manières de concevoir, exploiter et maintenir des systèmes urbains présents dans des territoires à risque. Premièrement, une stratégie possible dans la conception peut, en effet, consister en la protection des composants agressifs. L'objectif vis-à-vis de ces composants est d'opter pour une stratégie de fiabilisation renforcée au vu de leur rôle d'initiateur de multiples défaillances par effet 
domino. Cette fiabilisation peut passer par des mesures de surélévation (voies ferrées ou voies routières surélevées par exemple) ou de cloisonnement telles qu'elles permettent de minimiser les défaillances en chaîne (portes étanches dans les gares souterraines, les tunnels, par exemple). Deuxièmement, une stratégie dans le cadre de l'exploitation peut consister à assurer un suivi accru de l'état des composants au profil propagateur pour prévenir leur dysfonctionnement. La surveillance, à l'aide de capteurs de niveaux d'eau, des tunnels en tant que composant majoritairement diffuseur, est une stratégie usuellement mise en place sur les réseaux ferrés et routiers. Troisièment, une stratégie envisageable, pour la maintenance, peut être celle de considérer les composants sensibles comme ayant une durée de vie particulièrement limitée et devant donc faire l'objet d'un remplacement plus fréquent que les autres éléments du système.

\section{Conclusion}

Toutes les villes font face à des risques de typologie différente et d'intensité variable. Cette diversité des risques - naturels, technologiques, etc. - laisse apparaitre un trait commun qui est celui de la complexité des territoires urbains. Cette complexité, ou plus précisément cette haute complication, peut être appréhendée en partie par l'analyse des systèmes techniques qui constituent les villes : réseaux de transport, réseau d'alimentation en eau potable, etc. Ces infrastructures sont des éléments-clés de la maîtrise et de la gestion des risques urbains car ils sont d'une part vulnérables, et d'autre part, le bon fonctionnement d'une ville dépend du bon fonctionnement de ceux-ci.

Basée sur plusieurs méthodes issues de la sûreté de fonctionnement c'est-à-dire visant à étudier le fonctionnement et les dysfonctionnements potentiels d'un système, une démarche méthodologique globale permet la production des scénarios de défaillances par effet domino des composants d'un système lorsqu'il est touché par une inondation. La connaissance exhaustive de ces défaillances en chaîne est essentielle car elles sont un facteur dominant de la vulnérabilité d'un système. C'est donc cette démarche d'analyse interne du système qui donne une caractérisation de la vulnérabilité, nécessaire mais non suffisante, à toute amélioration de la résilience.

Appliquée aux systèmes de transport guidé subissant une inondation, cette méthodologie apparaît tout à fait applicable aux autres systèmes urbains au vu des structures et des fonctions similaires qu'ils présentent : systèmes de transport routier, de production d'eau potable, de gestion des eaux usées, etc. Tous ces systèmes ont, en effet, une architecture et une organisation similaires qui permet l'application, dans le principe au moins, d'une démarche méthodologique identique. Néanmoins, la méthode jusqu'ici particulièrement théorique nécessite des développements d'ordre opérationnel afin de devenir un outil d'aide à la décision en termes de conception et d'exploitation des systèmes urbains par les exploitants et les gestionnaires. Parmi ces développements, la confrontation des résultats de la méthode à des incidents passés sur les systèmes urbains apparaît essentielle afin d'améliorer celle-ci par la validation de défaillances par effet domino mises en évidence de façon théorique par la démarche méthodologique, par le retour d'expériences de perturbations de systèmes urbains et des mesures prises pour le fiabiliser, etc. 


\section{Bibliographie}

[BAR 16] BARROCA, B., BETHELOT S. et LAFFRECHINE K. «Génie urbain : la réinvention permanente», Diagonal Revue des équipes d'urbanisme, Vol. Novembre, N 198, p. 21-23, 2016.

[BER 13] BERAUD H., Initier la résilience du service de gestion des déchets aux catastrophes naturelles - Le cas des territoires urbains et de l'inondation, Thèse de doctorat, Université Paris-Est, 2013.

[COM 13] COMMISSARIAT GENERAL AU DEVELOPPEMENT DURABLE, Approche intégrée de la résilience des territoires Séminaire de travail 5 février, Paris, 2013.

[DHS 06] DHS, National Infrastructure Protection Plan, Washington, D.C., 2006.

[EWE 12] EWENT project (Extreme Weather impacts on transport systems) (2010-2012). Funded by the 7th framework program of the European Commission, Directorate General for Research and Technical Development. Coordinated by Technical Research Centre of Finland (VTT). http://ewent.vtt.fi

[GON 16] Gonzva M. Barroca B., Lhomme S., Gautier P.-E., Diab Y., " Apport de la sûreté de fonctionnement à l'analyse spatialisée du risque inondation ", Revue Internationale de Géomatique - Numéro spécial "Innovations Géomatiques pour les Risques Naturels et Technologiques”, Juillet-Septembre, pp.329-361, 2016.

[GON 17] GonzVA, M.. Résilience des systèmes de transport guidé en milieu urbain: approche quantitative des perturbations et stratégies de gestion, Thèse de doctorat, Université Paris-Est, 2017.

[GOU 13] Gouadjo Kagho N.C., Etude de la vulnérabilité et de la robustesse des ouvrages, Thèse de doctorat, Université Paris-Est., 2013.

[GRO 15] GROENEMEIJER P., "Hazard Identification in the RAIN project" [Presentation PowerPoint]. RAIN Workshop on Past Severe Weather Hazards, RAIN Project, Berlin, 27 February, 2015.

[KRO 08] KRÖGER W., "Critical infrastructures at risk: A need for a new conceptual approach and extended analytical tools”, Reliability Engineering \& System Safety, Vol. 93, N¹2, pp.1781-1787, 2008.

[LAI 00] LAIR J. Evaluation de la durabilité des systèmes constructifs du bâtiment, Thèse de doctorat, Université BlaisePascal, 2000.

[LHO 10] LhOMme S., SerRe D., Diab Y., LAGANiER R., « Les réseaux techniques face aux inondations ou comment définir des indicateurs de performance de ces réseaux pour évaluer la résilience urbaine », Bulletin de l'Association de géographes français, pp.487-502, 2010.

[MOR 01] MORTUREUX Y., "La sûreté de fonctionnement: méthodes pour maîtriser les risques ». Techniques de l’Ingénieur, Vol. 33, 2001.

[MOW 14] MOWE-IT project (Management of Weather Events In the Transport system) (2012-2014). Funded by the 7th framework program of the European Commission. Coordinated by Technical Research Centre of Finland (VTT). http://www.mowe-it.eu

[NOY 07] NoYes D. \& PERES F., Analyse des systèmes - Sûreté de fonctionnement. Techniques de l’Ingénieur, Vol. 33, 2007.

[NEL 07] NELSON, D. R., W. N. ADGER et K. BROWN. « Adaptation to Environmental Change : Contributions of a Resilience Framework », Annual Review of Environment and Resources, Vol. 32, N¹, 2007.

[PAR 08] PARLEMENT EuROPEen, Directive 2008/114/CE du 8 décembre 2008 concernant le recensement et la désignation des infrastructures critiques européennes ainsi que l'évaluation de la nécessité d'améliorer leur protection, 2008.

[PEY 02] PEYRAS L., Diagnostic et analyse de risques liés au vieillissement des barrages - Développement de méthodes d'aide à l'expertise, Thèse de doctorat, Université Blaise Pascal - Clermont II, 2002.

[PSC 09] PSC, National Strategy for Critical Infrastructure, Canada, 2009.

[SER 05] SERRE D., Evaluation de la performance des digues de protection contre les inondations - Modélisation de critères de décision dans un Système d'Information Géographique, Thèse de doctorat, Université de Marne-la-Vallée, 2005.

[SER 11] SERRE D., La ville résiliente aux inondations - Méthodes et outils d'évaluation, Mémoire d'Habilitation à Diriger des Rercherches, Université Paris-Est, 2011. 
[SER 15] SERRE, D. 2015b, «Concevoir la résilience urbaine : un défi face à des complexités», dans Complexité et désordre - Éléments de réflexion, Édité par EDP Sciences.

[SIM 91] SIMON H.A., Sciences des systèmes, sciences de l'artificiel, Dunod, Paris, 1991.

[TAL 06] TALON A., Evaluation des scénarii de dégradation des produits de construction, Thèse de doctorat, Université Blaise Pascal - Clermont II, 2006.

[TAL 09] Talon A., Boissier D., Peyras L., Démarches d'analyse de risques - Méthodes qualitatives d'analyse de risques. Université Numérique Ingénierie et Technologie (UNIT), 2009.

[TOU 14] TOUBIN M., Améliorer la résilience urbaine par un diagnostic collaboratif - L'exemple des services urbains parisiens face à l'inondation, Thèse de doctorat, Université Paris-Diderot, 2014.

[VAL 03] VAladAs E., Identification des scénarios de dégradations des chaussées bitumineuses épaisses, Thèse de docotrat, Université Blaise-Pascal, 2003.

[VIL 97] VILLEMEUR A., Sûreté de fonctionnement des systèmes industriels - Fiabilité, facteurs humains, informatisation Eyrolles, Paris, 1997.

[VUI 12] VUILLET M., Élaboration d'un modèle d'aide à la décision basé sur une approche probabiliste pour l'évaluation de la performance des digues fluviales, Thèse de doctorat, Université Paris-Est Marne-la-Vallée, 2012.

[ZWI 96] ZWINGELSTEIN G., La maintenance basée sur la fiabilité, Hermes Sciences, Paris, 1996.

[ZWI 09] ZWINGELSTEIN G., «Sûreté de fonctionnement des systèmes industriels complexes - Analyse prévisionnelle et bases de données de fiabilité », Techniques de l’ingénieur, Juin, 2009. 